\title{
On the Microwave Photonics Based Pulsed-Time-of- Flight Techniques in the Measurement of the Thickness of Dielectric Sheets
}

\author{
Janne P. Aikio, Juha Kostamovaara \\ Circuits and Systems Research Unit \\ UNIVERSITY OF OULU \\ Oulu, Finland \\ janne.aikio@oulu.fi
}

\author{
Markus Berg, Erkki T. Salonen \\ Centre for Wireless Communications \\ Radio Technologies Research Unit \\ UNIVERSITY OF OULU \\ Oulu, Finland
}

\begin{abstract}
This paper proposes a time domain measurement technique for characterizing the thickness of dielectric material based on pulse-domain microwave photonics and pulsed time-offlight configuration. Short laser pulses from a semiconductor laser diode are converted to electrical pulses using a wideband photodetector. These pulses are fed to an antenna, providing a simple and accurate non-destructive measurement technique for material characterization. The thickness of the sample is calculated based on the propagation delay of a radio wave by measuring the transit time of a short electromagnetic pulse. The propagation delay is determined from the front edge of the energy envelope. Results show that the measured propagation delay is linearly dependent on the sample thickness. The technique achieves a measurement precision in the sub-mm range with a measurement time of $40 \mathrm{~ms}$.
\end{abstract}

Keywords-Pulsed time-of-flight method; microwave photonics; non-destructive techniques; propagation delay

\section{INTRODUCTION}

Microwave measurements are preferred in various applications such as defining material properties [1], microwave radars [2], measurement of the frequency response of RF and millimeter wave circuits and components, and characterizing radio channels [3]. This is due to the good penetration properties of microwaves. In addition, the available bandwidth (BW) of the commercial measurement equipment, mainly the Vector Network Analyzer (VNA) is up to $100 \mathrm{GHz}$, which allows accurate measurements circuit and systems with sub-mm precision. The main difficulty with continuous wave (CW)based measurements, however, is the generation of continuous high power signals at a frequency of tens of gigahertz. This is a serious problem, since losses in dielectric materials, free space or long cables rapidly increase as a function of frequency. Similar problems apply to the generation of electromagnetic (EM) pulses, which can be used instead of CW signals.

In this paper we will study the possibility of using pulsedomain microwave photonics to generate short, intense ultrawide-band (UWB) pulses, which are then used in a pulsed timeof-flight configuration to characterize the thickness of dielectric samples. The main benefit of the proposed pulsed time-of-flight approach is that it is relatively simple and requires a minimum amount of signal processing but still allows quite accurate thickness determination based on timing of the front edge of the energy envelope of the transmitted pulse. Rather than presenting a method for immediate applications, we will study the performance of the proposed approach and present a simple transmitter configuration with extensive measurements.

This paper is organized as follows. A brief overview of sample thickness measurement is provided in Section II, the basic principle of the microwave photonics-based pulsed timeof-flight approach is presented in Section III, the measurement setup is explained in Section IV and the results are shown in Section V. Conclusions are given in Section VI.

\section{SAMPle Thickness MEASUREMENT}

When measuring sample thickness, non-invasive, nondestructive and contactless techniques are preferred in cases where the object to be measured is hot, inaccessible, or in motion. Non-destructive techniques (NDT) for thickness measurement [4] [5], such as radiography [6][7], ultrasonics [8], eddy-current [9], capacitive [10], inductive [11], microwave [1], open resonator [12] and optical [13] techniques, are presented in Table 1.

In general, optical techniques such as the optical interferometer, as in [13], or simple propagation delay measurement are superior when measuring transparent materials such as glass or plastics, for example. These techniques fail, however, when non-transparent materials are to be measured. Hence, microwave techniques are an obvious alternative, as they also provide good penetration properties with non-transparent materials. Since measurement resolution is inversely proportional to frequency bandwidth (BW), a BW of tens of $\mathrm{GHz}$ is required for sub-mm precision.

The proposed concept is the following. First, a laser driver and laser diode are used to produce short, high amplitude optical pulses in a range of 50-100ps. Then a wideband photodetector generates short, high amplitude electrical pulses that are fed to an antenna for free space measurement. After this the pulsed time-of-flight configuration, material thickness and permittivity can be calculated. The advantage of using laser pulses is that it enables easy, low loss propagation at longer distances via an optical fiber. 
TABLE I

NON-DESTRUCTIVE TECHNIQUES FOR MATERIAL CHARACTERIZATION

\begin{tabular}{|c|c|c|c|}
\hline Method & Operation principle & Advantages & Disadvantages \\
\hline Radiography [6],[7] & Ionizing radiation (e.g. X-rays) flux & $\begin{array}{l}\text { Versatile } \\
\text { Accurate }\end{array}$ & $\begin{array}{l}\text { Cost, Radioactive sources, } \\
\text { Shielding required }\end{array}$ \\
\hline Ultrasonic [8] & $\begin{array}{l}\text { Specific speed of sound in each material. } \\
\text { The propagation delay is measured. }\end{array}$ & $\begin{array}{l}\text { Low frequency } \\
\text { Low cost, Versatile }\end{array}$ & $\begin{array}{l}\text { Contact required } \\
\text { Lossy materials }\end{array}$ \\
\hline Eddy Current [9] & $\begin{array}{l}\text { High frequency AC current applied to a sensing } \\
\text { coil causes a magnetic field that induces an } \\
\text { Eddy current in the target material }\end{array}$ & $\begin{array}{l}\text { Large range and BW, Low cost } \\
\text { Tolerance of dirty environments }\end{array}$ & $\begin{array}{l}\text { Not suitable for thin materials } \\
\text { Sensors need to be close }\end{array}$ \\
\hline Capacitive [10] & $\begin{array}{l}\text { Thickness relative to the measured voltage (via } \\
\text { electric field) }\end{array}$ & $\begin{array}{l}\text { Low cost } \\
\text { Sub-nm resolutions }\end{array}$ & $\begin{array}{l}\text { Requires clean environment } \\
\text { Sensors need to be close }\end{array}$ \\
\hline Microwave [1] & $\mathrm{CW}$ measurements using scattering parameters & $\begin{array}{c}\text { Resolution } \\
\text { Penetration in non-metallic materials }\end{array}$ & $\begin{array}{l}\text { Wide BW for high resolution } \\
\text { Cost }\end{array}$ \\
\hline $\begin{array}{l}\text { Open Resonator Method } \\
\text { [12] }\end{array}$ & $\begin{array}{l}\text { Opposite concave mirrors form resonant modes } \\
\text { that are used to measure the sample }\end{array}$ & $\begin{array}{l}\text { Accurate determination of loss } \\
\text { tangent and } \epsilon_{r}\end{array}$ & $\begin{array}{l}\text { Complex and expensive test } \\
\text { fixture }\end{array}$ \\
\hline Optical Interferometer [13] & $\begin{array}{c}\text { A LED transmits light, and thickness is } \\
\text { measured based on reflections from each } \\
\text { surface }\end{array}$ & $\begin{array}{l}\text { Accurate, Repeatable } \\
\text { Measuring speed }\end{array}$ & $\begin{array}{l}\text { Not suitable for non-transparent } \\
\text { materials }\end{array}$ \\
\hline $\begin{array}{l}\text { Pulsed time-of-flight } \\
\text { (this work) }\end{array}$ & $\begin{array}{l}\text { Propagation delay measurement in a near field } \\
\text { using a short EM pulse and detection of the } \\
\text { front edge of the energy envelope }\end{array}$ & $\begin{array}{l}\text { Resolution, instrumentation } \\
\text { Penetration in non-metallic and non- } \\
\text { transparent materials } \\
\text { Simple and cost-efficient }\end{array}$ & $\begin{array}{l}\text { Generation of short, high } \\
\text { amplitude EM pulses } \\
\text { Difficult to calibrate }\end{array}$ \\
\hline
\end{tabular}

\section{A. Wave Propagation in a Medium}

The propagation velocity $v$ of a radio wave in a medium follows the equation

$$
v=f \lambda=\frac{1}{\sqrt{\mu_{r} \mu_{0} \epsilon_{r} \epsilon_{0}}}
$$

where $f=$ frequency, $\quad \lambda$ =wavelength,$\quad \mu_{r}=$ relative permeability and $\epsilon_{r}=$ relative permittivity of the medium. $\epsilon_{0}$ and $\mu_{0}$ are the permittivity and permeability in a vacuum. Since low loss materials are used here, only the real parts of $\epsilon_{r}$ and $\mu_{r}$ are taken into account. If the relative permittivity of the medium is known (non-magnetic medium assumed, i.e. $\mu_{r}=1$ ), the distance $d$ travelled by the wave can be calculated based on the propagation time: $d=v t$.

We can now calculate the wave propagation time in a free space proportional to the sample thickness $d$ and relative time delay as

$$
t_{0}=d \sqrt{\mu_{0} \epsilon_{0}},
$$

and the relative time delay including the sample as

$$
t_{r}=d \sqrt{\mu_{0} \epsilon_{r} \epsilon_{0}}
$$

The propagation delay in the medium is thus

$$
\begin{gathered}
t_{d}=t_{r}-t_{0}=d \sqrt{\mu_{0} \epsilon_{r} \epsilon_{0}}-d \sqrt{\epsilon_{0} \mu_{0}} \\
=d \sqrt{\mu_{0} \epsilon_{0}}\left(\sqrt{\epsilon_{r}}-1\right)
\end{gathered}
$$

which is linearly proportional to the thickness of the sample if the properties of the medium are constant. If $t_{d}$ can be measured and the properties of the medium are known, then the thickness of the sample can be determined from (4).
If the sample thickness and propagation delay are known, we can determine the relative permittivity $\epsilon_{r}$ by modifying (4), which yields

$$
\epsilon_{r}=\left(\frac{t_{d}}{d \sqrt{\epsilon_{0} \mu_{0}}}+1\right)^{2}
$$

\section{Measurement SetuP}

\section{A. Pulsed Time-of-Flight Using Microwave Photonics}

In the proposed method a short UWB pulse generated with a high speed laser diode and an optical detector working at microwave frequencies is transmitted through the sample and the corresponding transit time is measured. The test setup is illustrated in Fig 1. If part of the pulse energy is transmitted through a reference sample of known thickness, a differential transit-time measurement can reveal the thickness of the sample (with possible offset calibration), even if the dielectric constant of the material concerned is not known.

\section{B. Generation of the UWB Pulse}

Several electronic techniques for generating a UWB pulse are known, see [14], [15] and references therein. An alternative technique would be to use a sub-ns ( 100ps) high-energy laser pulse to illuminate a photodetector working at microwave frequencies. In this approach, the optical detector produces a current pulse, which can then be used to drive an antenna generating a UWB electro-magnetic pulse with an envelope closely matching the optical pulse shape [16]-[23]. The important advantage of this method is that the laser diode transmitter and the optical detector/antenna can easily be spatially separated with an optical fiber, considerably simplifying the transmitter configuration. As is well-known, this is not straight-forward for electric UWB pulses due to the large bandwidth and high center frequency, resulting in severe 


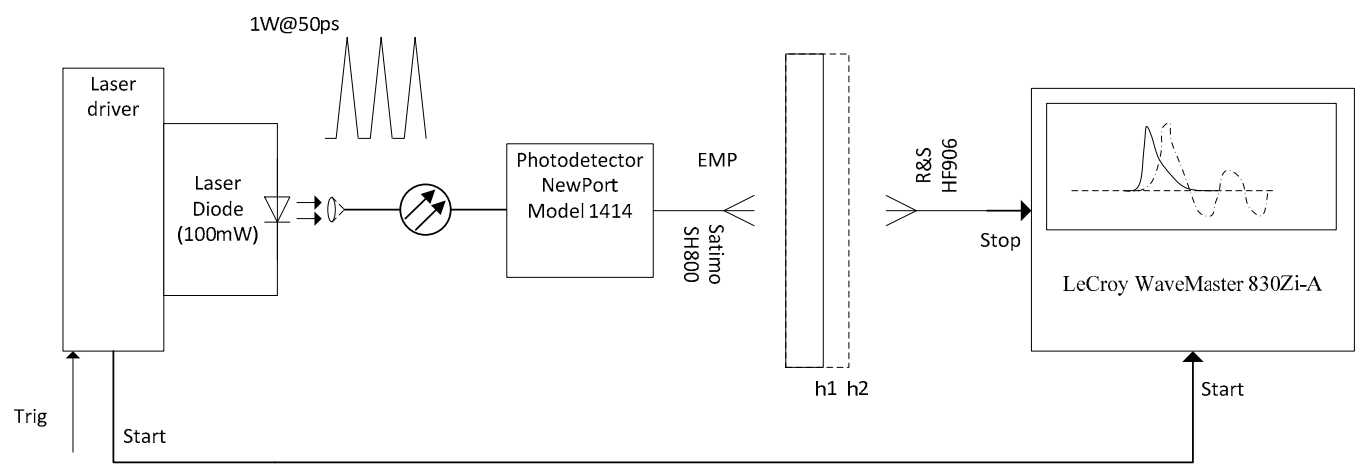

Fig. 1. Block diagram of the test setup for thickness measurement.

attenuation and pulse distortion in coaxial cables, for example. This approach, which is based on a combination of photonics and microwave techniques, has previously been applied for millimeter-wave (MMW) generation and WLAN communication systems, e.g. [16]-[23].

The UWB transmitter in our system is based on a semiconductor laser diode (LD) that operates in enhanced gain switching mode. Gain switching is a well-known method for producing isolated high-speed impulse-like pulses from a semiconductor laser diode (LD). It is based on a concept in which the internal resonator of the LD is brought into relaxation oscillations with a high-speed driving current edge. If the width of the driving current pulse is less than the distance between consecutive pulses of the relaxation oscillations, a single isolated pulse can in principle be achieved [24]. In the standard gain switching mode, however, the pulse energy is typically in the range of $\sim 10 \mathrm{pJ}$ only, and the trailing part of the pulse tends to show tailing.

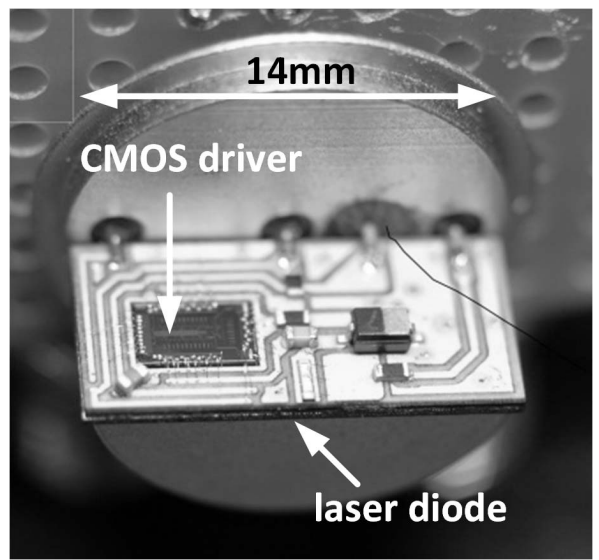

Fig. 2. Laser diode transmitter [26].

It has nevertheless been shown recently that the gain switching effect can be markedly enhanced by increasing the equivalent spot size (the thickness of the active region/confinement factor, $\mathrm{da} / \Gamma \mathrm{a}>>1)$ of the laser diode, resulting in notably higher optical power $(\sim 10 \mathrm{~W})$ and energy $(\sim 1 n J)$ [24], [25]. In the proposed measurement configuration a bulk laser diode working in the enhanced gain switching mode, giving a peak power of $\sim 2 \mathrm{~W}$ and driven by a full-custom CMOS driver, was used as an optical source (see Fig. 2 [26]). The optical output was conducted via an optical fiber to a high speed optical detector (NewPort Model 1414), which then fed its current output to the antenna.

\section{Free Space Propagation}

The free space propagation environment was formed by two ultra-wideband dual-ridge horn antennas: Satimo SH800 (transmitting) and Rohde \& Schwartz (R\&S) HF906 (receiving). These were located against each other at a distance of $d=550$ $\mathrm{mm}$ between the antenna connectors as shown in Fig. 3. The frequency response of the measured channel using $20 \mathrm{GHz}$ VNA is shown in Fig. 4, where it can be seen that the pass band of the channel is between $1-10 \mathrm{GHz}$, which is mainly determined by the pass band of the antennas.

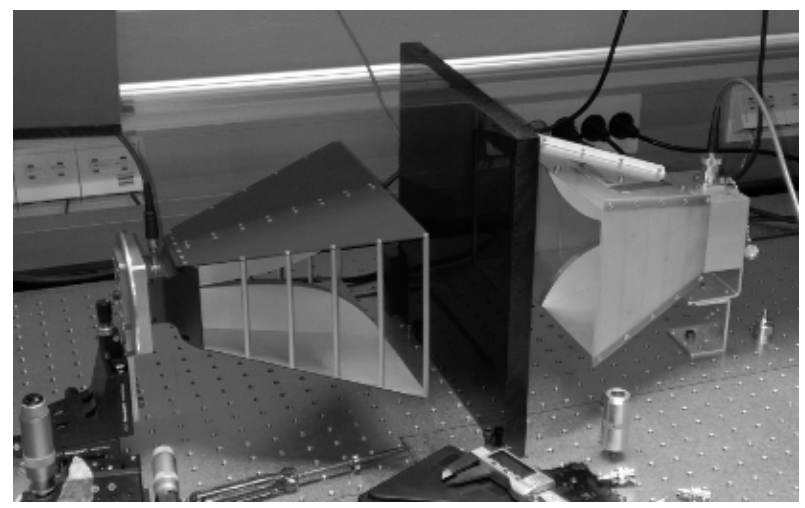

Fig. 3. Transmission measurement setup.

The shape of the transmitted pulse to be measured is presented in Fig. 5. The rise time of the pulse is around $60 \mathrm{ps,}$ the pulse width around $150 \mathrm{ps}$ and the peak amplitude given by the optical detector $1.5 \mathrm{~V}$ with a $50 \Omega$ antenna load. The shape of the received pulse without any dielectric samples is also presented in Fig. 5, where it can be seen that the received pulse is delayed (the reference transmit delay), and the shape is different from that of the transmitted pulse. In fact, the transmitted pulse is differentiated as a result of the frequency response of the channel. As shown in Fig. 4, the measuring channel has a band pass character with a lower corner frequency of around $1 \mathrm{GHz}$, which is caused by the bandwidth limitation of the antennas. The antennas cause frequency dispersion, i.e. phase delay in various frequency components resulting from the 
antenna geometry. Frequency dispersion and the band pass response of the channel are the reasons for the resonance after the received pulse (see the ringing after $2 \mathrm{~ns}$ of the received pulse in Fig. 5).

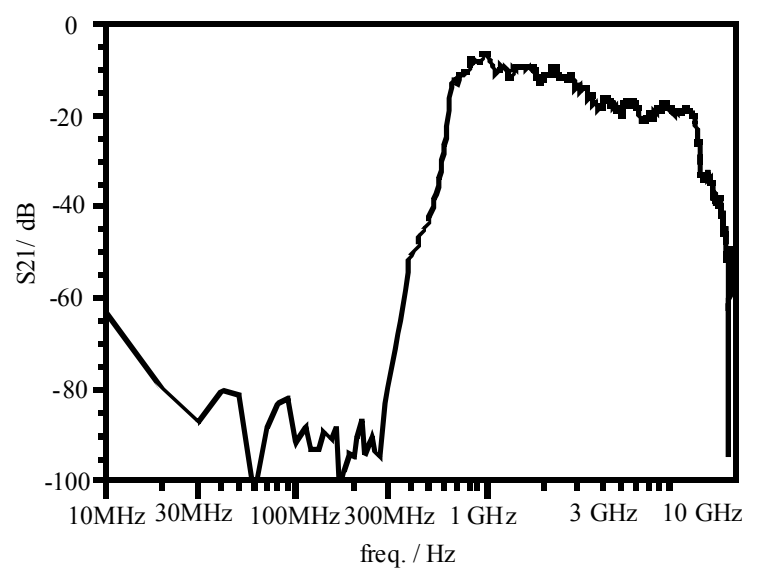

Fig. 4. Frequency response of the measuring channel, including antennas.

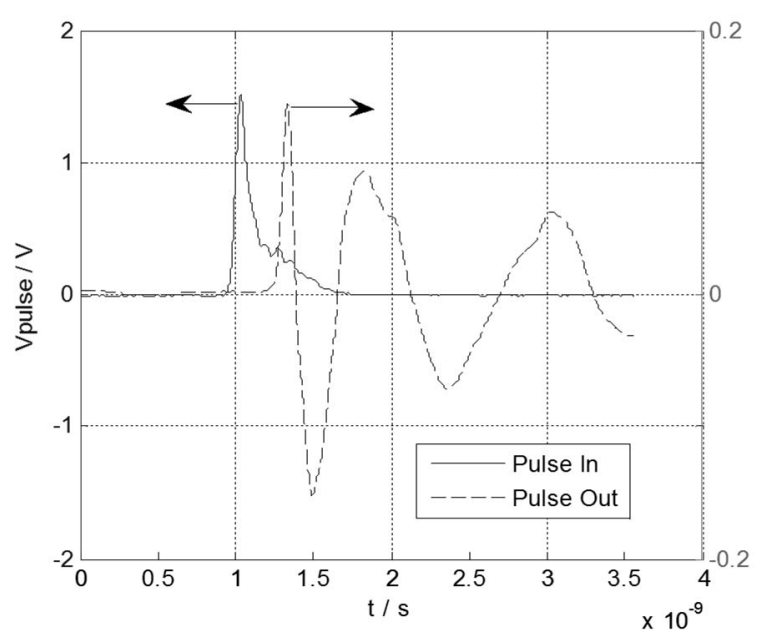

Fig.5. Pulse from the optical detector to a $50 \mathrm{Ohm}$ load (a), and through the transmission system to a $50 \mathrm{Ohm} \mathrm{load} \mathrm{(b).}$

The time interval measurements were performed using a LeCroyWaveMaster 830Zi-A Oscilloscope. The measurement procedure was the following. The pulse rate of the pulse generator was set to $10 \mathrm{kHz}$, and the oscilloscope measured the time delay between the transmitted pulse and the reference pulse. The amplitude value of the steepest slope of the front edge envelope of the received pulse was used. The total single shot jitter of the measurement is in the range of $5 \mathrm{ps}$, which was reduced below $1 \mathrm{ps}$ by averaging 400 measurements. This corresponds to a measurement time of $40 \mathrm{~ms}$. Comparable measurements were performed using an R\&S ZVB 20 VNA in the time domain mode.

\section{Selected Dielectric Material}

The dielectric materials chosen for the characterization were polyethylene PE300 $\left(\epsilon_{r}=2.5\right)$ and $\operatorname{PE500}\left(\epsilon_{r}=2.4\right)$ manufactured by Roechling. 12 sample sheets of PE300 and four of PE500 were available for the measurements. The size of each sample was $300 \mathrm{~mm}$ x $300 \mathrm{~mm}$, and the thicknesses are shown in Table II. Each sample was measured separately with a slide gauge, although, as the wave propagates at the center of the sample, the thickness measured at the edge may not be reliable. In order to verify the measured thickness we calculated the mean thickness $h$ of each sample by

$$
h=\frac{m}{\mathrm{w} \cdot l \cdot \gamma}
$$

where $m=$ mass, $w=$ width, $l=$ length and $\gamma=$ density of the medium, which in this case was $\gamma=0.96 \mathrm{~g} / \mathrm{cm}^{3}$ for both the PE300 and PE500 samples. The values obtained from (6), as used in this paper, are presented in Table II.

TABLE II

THICKNESSES OF THE SAMPLES

\begin{tabular}{cccccc}
\hline \hline PE & Thickness & PE & Thickness & PE & Thickness \\
300 & & 300 & & 500 & \\
\hline$h_{1}$ & $3.97 \mathrm{~mm}$ & $h_{7}$ & $6.12 \mathrm{~mm}$ & $h_{13}$ & $10.07 \mathrm{~mm}$ \\
$h_{2}$ & $4.91 \mathrm{~mm}$ & $h_{8}$ & $9.92 \mathrm{~mm}$ & $h_{14}$ & $20.07 \mathrm{~mm}$ \\
$h_{3}$ & $4.91 \mathrm{~mm}$ & $h_{9}$ & $9.95 \mathrm{~mm}$ & $h_{15}$ & $20.10 \mathrm{~mm}$ \\
$h_{4}$ & $4.94 \mathrm{~mm}$ & $h_{10}$ & $10.03 \mathrm{~mm}$ & $h_{16}$ & $20.05 \mathrm{~mm}$ \\
$h_{5}$ & $6.01 \mathrm{~mm}$ & $h_{11}$ & $20.13 \mathrm{~mm}$ & & \\
$h_{6}$ & $6.09 \mathrm{~mm}$ & $h_{12}$ & $30.56 \mathrm{~mm}$ & & \\
& & & & & \\
\hline \hline
\end{tabular}

\section{MEASUREMENT RESUlts}

The recorded time-domain waveforms for each measurement of the PE300 samples are shown in Fig. 7. A total of 40 measured channel impulse responses are presented. Each curve represents a different PE300 sample thickness between 0 and $85 \mathrm{~mm}$. Note that the samples were stacked in order to obtain a gradual increase in thickness. The propagation delays were determined between the maximum amplitudes of the impulse responses. It is easy to distinguish the reference waveform $h_{0}$ (no sample) in Fig. 7, as it is the first waveform, that with the highest amplitude. Note that the peak amplitude of the samples gradually increases as a function of thickness. As the transmitted power is constant, the increased amplitude refers to increased directivity due to the larger volume of dielectric material in the near field of the antenna.

The propagation delays are shown as a function of sample thickness for PE300 and PE500 using pulsed measurements in Fig. 8. It can be seen that the measured propagation delays vs. sample thickness are linear in both materials. The difference in $\epsilon_{r}$ between PE300 and PE500 causes a variation in slope, while the difference between the measured and calculated results is due to the fact that the $\epsilon_{r}$ provided by the sample supplier is characterized at $1 \mathrm{MHz}$ while our characterization was performed in the $\mathrm{GHz}$ range.

In order to illustrate the achieved precision more clearly the measured propagation delay vs. the sample thickness of PE300 is presented in Fig. 9 for sheet thicknesses between 4 to $13 \mathrm{~mm}$, showing some variation compared with the reference result. These results suggest that the precision achieved in this setup is around $+/-2 \mathrm{ps}$, corresponding to $\sim+/-0.3 \mathrm{~mm}$. This precision was achieved with a measurement time of $\sim 40 \mathrm{~ms}$. The transmitter 
allows the pulse rate to be increased to $\sim 1 \mathrm{MHz}$, which would increase the measurement speed correspondingly.

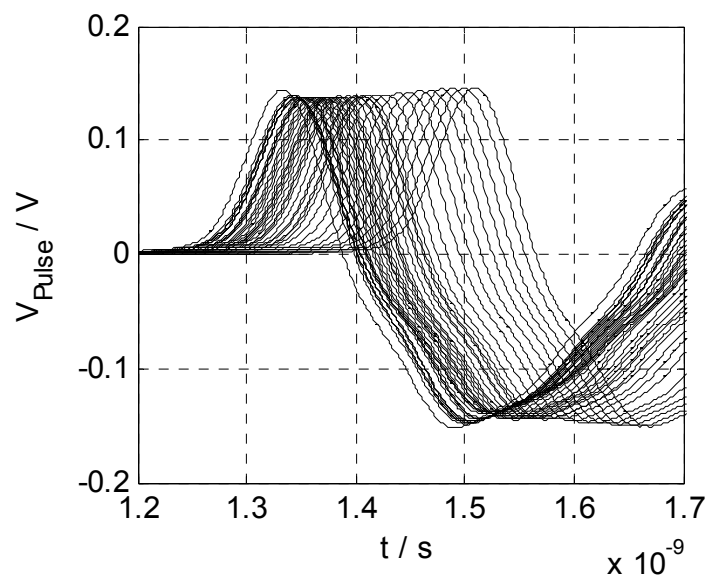

Fig. 7. Received pulses at sample (PE300) thicknesses from $0 \mathrm{~mm}$ to $85 \mathrm{~mm}$.

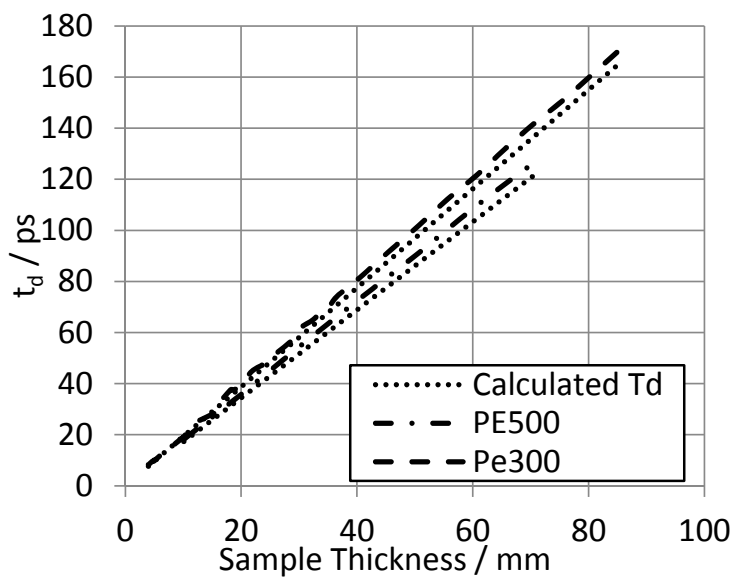

Fig. 8. Propagation delay $t_{d}$ vs. sample thickness of PE500 and PE300 using pulsed time-of-flight measurements and calculated ideal $t_{d}$.

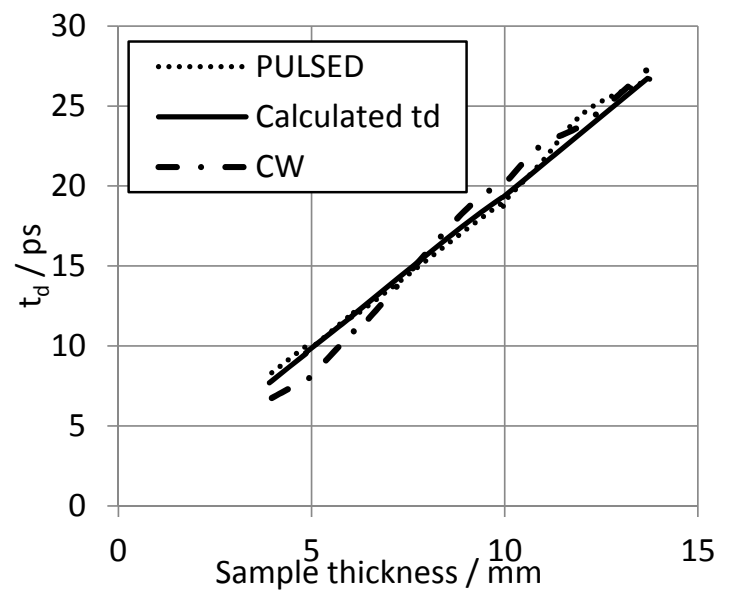

Fig. 9. Propagation delay $t_{d}$ vs. sample thickness of PE300 and calculated ideal $t_{d}$ using pulsed and $C W$ measurements.
In addition to enabling characterization of the sheet thickness, the proposed method also allows the characterization of the dielectric constant for known sheet thicknesses. As a demonstration of this, the relative permittivity of the PE300 $\left(\epsilon_{r}=2.5\right)$ and PE500 $\left(\epsilon_{r}=2.4\right)$ samples were calculated and compared using the propagation delay differences for the various sample thicknesses. The results are shown in Fig. 6 and Fig. 10 as a function of sample thickness. The inaccuracy limits with regard to the measured permittivity caused by the time resolution of 1 ps are also shown in Fig. 6. It should be noted that the time domain method indicates $\epsilon_{r}$ at the higher corner frequency of the bandwidth (see Fig. 4), whereas the sample supplier provided values at $1 \mathrm{MHz}$.

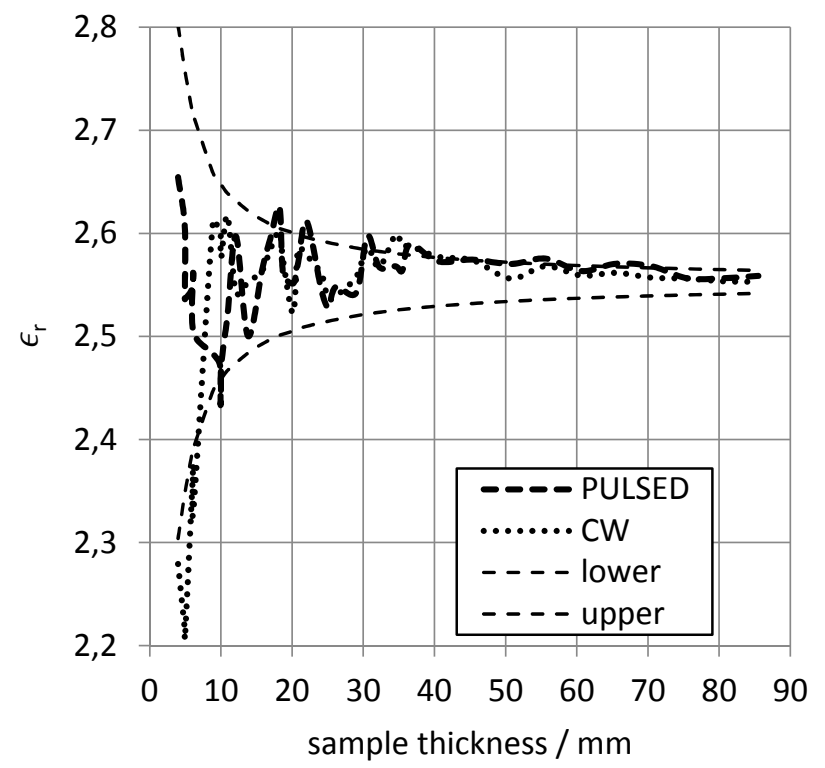

Fig. 6. Relative permittivity of PE300 as calculated from the measured results as a function of sample thickness, including the upper and lower inaccuracy limits for both measurements caused by the time resolution of $1 \mathrm{ps}$.

It can be seen from Fig. 6 that the measured permittivity varies considerably when the sample thickness is less than $\sim 10$ $\mathrm{mm}$ and begins to stabilize as the sample thickness increases. The relative permittivity of PE500 as a function of sample thickness is shown in Fig. 10. As implied in Table II, only 4 samples of PE500 were used, and therefore fewer measurement points were available. Fortunately, samples $h_{14}, h_{15}$ and $h_{16}$ were cut from the same larger sheet, which means that variations within these samples are minimized. Indeed, as seen in the figure, $\epsilon_{r}$ is quite stable above $20 \mathrm{~mm}$.

\section{CONCLUSIONS}

We have demonstrated here the capability of microwave photonics for characterizing the sample thickness of dielectric material. The proposed measurement technique employs the pulsed time of flight method by generating a UWB pulse using a laser diode working in the enhanced gain switching mode and a high-speed optical detector. The technique easily allows a relatively large distance between the laser diode transmitter and the optical detector/antenna. 
In the present case a specialized high-speed oscilloscope was used for the time interval measurements. It is known, however, that high performance time-to-digital converters with up to $\sim 5 \mathrm{ps}$ single shot precision can be realized with CMOS techniques [27]. Thus the system realization can be miniaturized to a considerable extent by using it together with a laser transmitter micro-module.

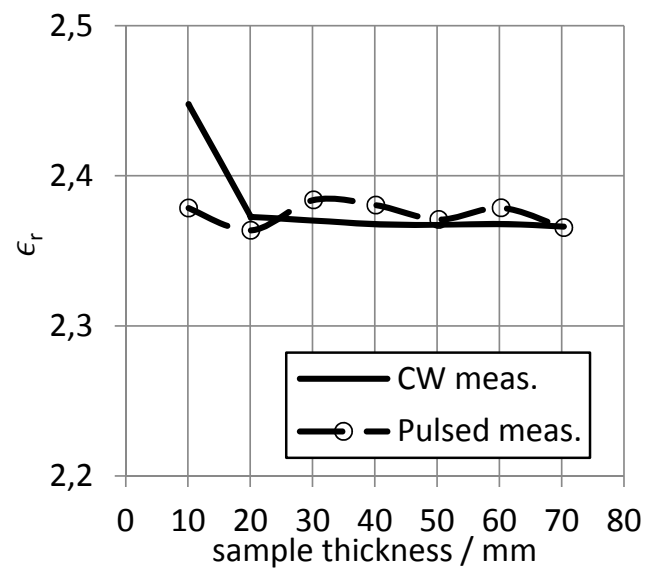

Fig. 10. Relative permittivity of PE500 vs. sample thickness.

\section{REFERENCES}

[1] U. C. Hasar, O. Simsek "On the application of microwave calibrationindependent measurement for noninvasive thickness evaluation of medium- or low-loss solid materials" Progress In Electromagnetics Research, PIER 91, 377-392, 2009.

[2] I. Gresham; A. Jenkins; R. Egri; C. Eswarappa; N. Kinayman; N. Jain; R. Anderson; F. Kolak; R. Wohlert; S. P. Bawell; J. Bennett; J. -P. Lanteri, "Ultra-wideband radar sensors for short-range vehicular applications", IEEE Tran. Microw. Theory and Tech. pp. 2105 - 2122, vol. 52, no.9, Sept. 2004.

[3] A. Roivainen, C. F. Dias, N. Tervo, V. Hovinen, M. Sonkki, and M. Latva-aho, "Geometry-Based Stochastic Channel Model for Two-Story Lobby Environment at $10 \mathrm{GHz}$ ", IEEE Tran. on Antennas and propagation, pp. 3990-4003, vol. 64, no. 9, Sept. 2016.

[4] J. C. Brasunas, G. M. Cushman and B. Lakew, "Thickness measurement" in Measurement, Instrumentation, and Sensors Handbook, J. G. Webster and H. Eren ed. Florida: CRC Press, 2014, pp. 13.1-13.10

[5] Blitz, J., Electrical and Magnetic Methods of Non-destructive Testing, Chapman \& Hall, London, UK, 1997.

[6] K. Iguchi, "Thickness Gages for Films and Sheets Extrusion Lines," J. of Plastics, vol. 47, no. 4, 1996, pp. 87-94

[7] A. T. Macrander, S. Lau, K. Strege, and S. N. G. Chu, "Nondestructive measurement of layer thickness in double heterostructures by X-ray diffraction,”Appl. Phys. Lett.,vol.52, pp.1985-1986, Apr., 1988.

[8] Jin-sheng Yang, Xiao-guang Zhao, Yong-jiang Zhang and Zhe Cao, "A new type of wheeled intelligent ultrasonic thickness measurement system" In Proc. Symposium on Piezoelectricity, Acoustic Waves and Device Applications (SPAWDA), 25-27 Oct. 2013.

[9] K. Suresh, G. Uma, M. A. Umapathy, "New Resonance-Based Method for the Measurement of Nonmagnetic-Conducting-Sheet Thickness" IEEE Trans. on Instrumentation and measurement. Vol. 60, pp. 3892 3897, 2011.

[10] T. O. Kim, H. Y. Kim, C. M. Kim, and J. H. Ahn, "Non-contact and inprocess measurement of film coating thickness by combining two principles of eddy-current and capacitance sensing" Ann. CIRP , vol. 56, no. 1, pp. 509-512, 2007.
[11] Y. Nonaka, "A double coil method for simultaneously measuring the resistivity, permeability, and thickness of a moving metal sheet" IEEE Trans. on Instrumentation and measurements vol. 45, no. 2, pp. 478-482, Apr. 1996.

[12] T. Hirvonen, P. Vainikainen, A. Lozowski, A. Räisänen, ” Measurement of dielectrics at $100 \mathrm{GHz}$ with an open resonator connected to a network analyzer", IEEE Trans. on Instrum.Meas., Vol 45, no.4, pp. 780-786, Aug. 1996.

[13] J. Eom, S. Jun Park, Y. Ho Kim, B. Ha Lee, "Non-destructive and noncontact thickness measurement for optically opaque samples by optical fiber heterodyne interferometry system" In Proc. Lasers and ElectroOptics Pacific Rim (CLEO-PR) Conference, 2013.

[14] M. Fanbao, Z. Chuanming, Y. Zhoubing, J. Bingquan, W. Wentao and M. Hongge, "UWB pulse generation and radiation using photoconductive switching in GaAs:Cr", Proc. IEEE Int. Symp. Discharges Electrical Insulation in Vacuum. pp. 785-787, 1998.

[15] H. Zhang, X. Liu, N. Li and T.A. Gulliver, "A novel UWB pulse generator", Communications Computers and Signal Processing (PacRim) 2011 IEEE Pacific Rim Conference On. IEEE, pp. 691-695, 2011.

[16] M. Malekizandi, Q. T. Le, D. Briggmann, A. Emsia and F. Küppers, "Radio transmission and BER performance of UWB pulse generation based on directly modulated semiconductor laser" In Proc. IEEE conference on Optical Interconnects (OI), pp. 74-75, 2015.

[17] S. Pan and J. Yao, "UWB-Over-Fiber Communications: Modulation and Transmission", Journal of Lightwave Technology, vol. 28, pp. 2445-2455, 2010.

[18] Q. T. Le, D. Briggmann and F. Ktippers, "Generation of UWB pulses using direct modulation of semiconductor laser and optical filtering", Electronics Letters, vol. 49, pp. 1171-1173, August 2013.

[19] H. Shams, A. Kaszubowska-Anandarajah, P. Perry, P. Anandarajah, and L. Barry, "Electro-Optical Generation and Distribution of Ultra-wideband Signals Based on the Gain Switching Technique," Journal of Optical Communications and Networking, vol. 2, no. 3, pp. 122-130, 2010.

[20] Y. Yashchyshyn, A. Chizh, A. Urzedowska, S. Malyshev and J. Modelski, "Transmitting and receiving photonic antennas for wireless LAN", Proc. EuMW'10, pp. 129-132, 2010.

[21] A. Chizh; S. Malyshev; S. Jefremov; B. Levitas; I. Naidionova, "Impulse transmitting photonic antenna for ultra-wideband applications" In Proc.18th International Conference on Microwave Radar and Wireless Communications (MIKON), 2010.

[22] B. Levitas, M. Drozdov, I. Naidionova, S. Jefremov, S. Malyshev and A. Chizh, "UWB system for time-domain near-field antenna measurement", Proc. EuMW'13, pp.388 - 391, 2013.

[23] J. Yao, F. Zeng, Q, Wang, "Photonic Generation of Ultrawideband Signals", Journal of Lightwave Technology, pp. 3219 - 3235, vol. 25, no. 11, Nov. 2007.

[24] B. Ryvkin, E. Avrutin, J. Kostamovaara, "Asymmetric-waveguide laser diode for high-power optical pulse generation by gain switching", IEEE/OSA Journal of Lightwave Technology in 2009, Volume 27, Issue 12, 2009, pp. 2125 - 2131.

[25] B. Ryvkin, E. A. Avrutin and J. T. Kostamovaara, "Quantum Well laser with an extremely large active layer width to optical confinement factor ratio for high-energy single picosecond pulse generation by gain switching”, Semicond.Sci. and Tecnology, vol. 26, no. 4, 10p, 2011

[26] J. Nissinen, J. Kostamovaara, "A 1 A laser driver in 0.35 um complementary metal oxide semiconductor technology for pulsed timeof-flight laser rangefinder" Review of Scientific Instruments 80(10): 104703, 2009.

[27] P. Keränen and J. Kostamovaara, "A Wide Range, 4.2 ps(rms) Precision CMOS TDC With Cyclic Interpolators Based on Switched-Frequency Ring Oscillators," in IEEE Transactions on Circuits and Systems I: Regular Papers, vol. 62, no. 12, pp. 2795-2805, Dec. 2015. 